University of Nebraska - Lincoln

DigitalCommons@University of Nebraska - Lincoln

$11-1-2002$

\title{
Magnetic relaxation phenomena in the superspin-glass system $\left[\mathrm{Co}_{80} \mathrm{Fe}_{20} / \mathrm{Al}_{2} \mathrm{O}_{3}\right]_{10}$
}

\author{
Sarbeswar Sahoo \\ Laboratorium für Angewandte Physik, Gerhard-Mercator-Universität Duisburg, D-47048 Duisburg, \\ Germany, sarbeswar@gmail.com \\ O. Petracic \\ Laboratorium für Angewandte Physik, Gerhard-Mercator-Universität Duisburg, D-47048 Duisburg, Germany \\ Christian Binek \\ University of Nebraska-Lincoln, cbinek@unl.edu \\ Wolfgang Kleemann \\ Laboratorium für Angewandte Physik, Gerhard-Mercator-Universität Duisburg, D-47048 Duisburg, \\ Germany, wolfgang.kleemann@uni-due.de
}

J. B. Sousa

IFIMUP, Departamento de Fisica, Universidade de Porto, 4169-007 Porto, Portugal

See next page for additional authors

Follow this and additional works at: https://digitalcommons.unl.edu/physicsbinek

Part of the Physics Commons

Sahoo, Sarbeswar; Petracic, O.; Binek, Christian; Kleemann, Wolfgang; Sousa, J. B.; Cardoso de Freitas, Susana; and Freitas, P. P., "Magnetic relaxation phenomena in the superspin-glass system $\left[\mathrm{Co}_{80} \mathrm{Fe}_{20} / \mathrm{Al}_{2} \mathrm{O}_{3}\right]_{10 "}$ (2002). Christian Binek Publications. 35.

https://digitalcommons.unl.edu/physicsbinek/35

This Article is brought to you for free and open access by the Research Papers in Physics and Astronomy at DigitalCommons@University of Nebraska - Lincoln. It has been accepted for inclusion in Christian Binek Publications by an authorized administrator of DigitalCommons@University of Nebraska - Lincoln. 
Authors

Sarbeswar Sahoo, O. Petracic, Christian Binek, Wolfgang Kleemann, J. B. Sousa, Susana Cardoso de Freitas, and P. P. Freitas 


\title{
Magnetic relaxation phenomena in the superspin-glass system $\left[\mathrm{Co}_{80} \mathrm{Fe}_{20} / \mathrm{Al}_{2} \mathrm{O}_{3}\right]_{10}$
}

\author{
S. Sahoo ${ }^{1}$, O. Petracic ${ }^{1}$, Ch. Binek ${ }^{1}$, W. Kleemann ${ }^{1}$, J. B. Sousa ${ }^{2}$, \\ S. Cardoso ${ }^{3}$, and P. P. Freitas ${ }^{3}$ \\ ${ }^{1}$ Laboratorium für Angewandte Physik, Gerhard-Mercator-Universität Duisburg, D- \\ 47048 Duisburg, Germany \\ ${ }^{2}$ IFIMUP, Departamento de Fisica, Universidade de Porto, 4169-007 Porto, Portugal \\ ${ }^{3}$ INESC, Rua Alves Redol 9-1, 1000 Lisbon, Portugal
}

\begin{abstract}
Relaxation and temperature cycles of thermoremanent magnetization, $M^{\mathrm{TRM}}$, in the superspin-glass phase of $\left[\mathrm{Co}_{80} \mathrm{Fe}_{20}(0.9 \mathrm{~nm}) / \mathrm{Al}_{2} \mathrm{O}_{3}(3 \mathrm{~nm})\right]_{10}$ have been investigated. The relaxation of $M^{\mathrm{TRM}}$ exhibits ageing phenomena. In negative temperature cycles for temperature steps larger than $1 \mathrm{~K}$ the magnetic state is retrieved (memory effect) on returning to the measurement temperature. This property is independent of the application of a field step during intermediate cooling. In positive temperature cycles the relaxation is suppressed after temporary heating. The observations are discussed in the light of both the droplet and the hierarchical picture.
\end{abstract}

\section{Introduction}

The dynamics of interacting ferromagnetic nanoparticle systems has been a subject of extensive research during the last decades $[1,2]$. In numerous investigations the existence of a low-temperature collective glassy dynamics below the so-called spin-glass temperature, $T_{\mathrm{g}}$, has been evidenced for ensembles of nanoparticles due to significant dipole-dipole interaction, and randomness of particle positions and directions of anisotropy axes [2-7]. Typical spin-glass characteristics reflecting nonequilibrium properties such as ageing, memory and rejuvenation phenomena have been observed in DC magnetic relaxation and low-frequency AC susceptibility experiments in the superspin-glass (SSG) phase [6] of frozen ferrofluids [2, 4, 8].

The dynamics in the SSG phase is strongly dependent on its thermomagnetic history. The response function is governed by several parameters such as the rate at which the temperature is changed to attain the measurement temperature, $T_{\mathrm{m}}<T_{g}$, and the wait time, $t_{w}$, at $T_{\mathrm{m}}$ before the response function is measured. In particular it has been shown that the thermal history in a rather narrow temperature range close to $T_{\mathrm{m}}$ governs the non-equilibrium dynamics [9], while the thermal history at higher temperatures is irrelevant. 
In particular for monodisperse $\epsilon-\mathrm{Fe}_{3} \mathrm{~N}$ nanoparticles with significant dipolar interaction [4], it has been shown that the ageing and memory effects are similar to those of archetypical spin glasses, whereas some differences have been reported for other systems due to the wider energy barrier distribution despite their obvious collective behavior [8]. A completely satisfactory theoretical model has not yet been established, but there has been considerable progress as regards understanding the experimental results in the light of existing models [10, 11]. Monte Carlo simulations have been developed and are in progress in order to elucidate the abundance of experimentally derived properties [12].

In this paper we report on the relaxation of the thermoremanent magnetization, $M^{\mathrm{TRM}}$, in the discontinuous metal-insulator multilayer (DMIM) system $\left[\mathrm{Co}_{80} \mathrm{Fe}_{20}(0.9 \mathrm{~nm}) / \mathrm{Al}_{2} \mathrm{O}_{3}(3 \mathrm{~nm})\right]_{10}$. It has previously been shown that this nanoparticle system exhibits a collective SSG phase at temperatures below $T_{\mathrm{g}} \approx 43.6 \mathrm{~K}[5,7]$. We shall discuss our results in the light of both the hierarchical [10] and the droplet model [11]. We find that in negative temperature cycles the magnetic state can be retrieved on returning to the previous temperature. The application of a field step upon cooling does not destroy the memory effect. For positive temperature cycles this memory effect is lost. Instead the relaxation is accelerated upon heating. While the negative temperature cycle observations are consistent with both models, the asymmetry observed in positive temperature cycles contradicts the droplet picture and can only be explained within the framework of a hierarchical organization of metastable states [13].

\section{Experimental details}

The sample, $\left[\mathrm{Co}_{80} \mathrm{Fe}_{20}\left(t_{n}=0.9 \mathrm{~nm}\right) / \mathrm{Al}_{2} \mathrm{O}_{3}(3 \mathrm{~nm})\right]_{10}$, was prepared by Xe-ion-beam sputtering on a glass substrate [14]. High-resolution transmission electron microscopy (HRTEM) images on a related sample with nominal CoFe thickness, $t_{\mathrm{n}}=1.3 \mathrm{~nm}$, reveal that the CoFe forms isolated quasispherical nanoparticles of average diameter $\mathrm{d} \approx 3 \mathrm{~nm}$ and a log-normal size distribution width $\sigma \approx$ 2.7 .

Relaxation measurements of $M^{\mathrm{TRM}}$ have been performed by use of a commercial superconducting quantum interference device magnetometer (Quantum Design MPMS-5S). For zero-field measurements, and for zero-field-cooling experiments the remanent field of the superconducting coil and the Earth's magnetic field, $\left|\mu_{0} \mathrm{H}\right| \approx 0.05$ and $0.046 \mathrm{mT}$, respectively, were compensated to within an accuracy of a constant (positive) field $<0.03 \mathrm{mT}$. The experimental time window spans the range from about 1 to $10^{5} \mathrm{~s}$. Different relaxation curves have been recorded after cooling the sample using the protocols described as follows:

(1) For measuring the relaxation of $M^{\mathrm{TRM}}$, the sample is cooled in a constant field from $T=100 \mathrm{~K}>$ $T_{\mathrm{g}}$ to the measurement temperature, $T_{\mathrm{m}}<T_{g}$, where after a wait time $t_{w}$ the field is set to zero.

(2) In a temperature cycle protocol the sample is cooled in a constant field from $T=100 \mathrm{~K}$ to the measurement temperature $T_{\mathrm{m}}<T_{\mathrm{g}}$ and, after the field is set to zero, the relaxation of the $M^{\mathrm{TRM}}$ is measured immediately for a period $t_{1}$; then the temperature is rapidly changed by $\Delta T$ and the relaxation is subsequently measured for a period $t_{2}$; and finally the cycle is completed by returning to $T_{\mathrm{m}}$,where the relaxation is recorded for a period $t_{3}$ (see Figure 2 for an example).

\section{Results and discussion}

One of the most striking properties of the relaxation in interacting nanoparticle systems below $T_{\mathrm{g}}$ is the ageing behavior [2]. Figure 1 shows the relaxation of $M^{\mathrm{TRM}}$ at $T_{\mathrm{m}}=40 \mathrm{~K}$. The cooling field $\mu_{0} \mathrm{H}$ 
$=0.4 \mathrm{mT}$ was switched off after wait times, $t_{w}=10^{2}, 10^{3}$ and $10^{4} \mathrm{~s}$ following the above $M^{\mathrm{TRM}}$ protocol (1). In the inset we show the corresponding relaxation rate $S=(1 / H) \partial M / \partial \ln t$ versus $\ln t$. As can be seen, the wait time dependence is apparently reflected by the peak positions of $S, t_{p}=1000$, 1200 and $3000 \mathrm{~s}$, respectively, although their spread is less pronounced as compared to that of $t_{w}$. Probably this weak wait time dependence of the dynamics of our nanoparticle system when compared to an archetypical spin glass [15] can be assigned to the relatively wide distribution of particle sizes. The fraction of large particles undergoing a blocking transition at $T_{\mathrm{b}}>T_{\mathrm{g}}$ will not take part in collective dynamics. They exhibit an independent relaxational behavior whose peak relaxation seems to lie close to $10^{3} \mathrm{~s}$.

The temporal decay of $M^{\text {TRM }}$ can be approximately described by a stretched exponential function of the form

$$
M^{\mathrm{TRM}}=M_{0} \exp \left(-\left(t / \tau_{p}\right)^{1-n}\right)
$$

where $M_{0}$ and the response time $\tau_{p}$ do not depend on the observation time $t$ (but may depend on the temperature) and $n$ is a constant which depends on the wait time $t_{w}$ and temperature. Best fits of equation (1) to the $M^{\mathrm{TRM}}$ versus $\ln t$ data sets reveal: $\tau_{p}=1374 \pm 156 \mathrm{~s}$ and $n=0.79$ for $t_{w}=10^{2}$ $\mathrm{s} ; \tau_{p}=1727 \pm 158 \mathrm{~s}$ and $n=0.77$ for $t_{w}=10^{3} \mathrm{~s}$; and $\tau_{p}=3742 \pm 188 \mathrm{~s}$ and $\mathrm{n}=0.74$ for $t_{w}=10^{4} \mathrm{~s}$. Since $\tau_{p}$ designates the peak time of the relaxation rate $S$ as calculated from equation (1), its values are strongly correlated with the observed peak times $t_{p}$. Further, the slight decrease of $n$ with increasing $t_{w}$ seems to reflect the SSG ordering while waiting.

The ageing effects can be explained by the droplet model as follows. According to the droplet picture the approach towards equilibrium after a quench from above $T_{\mathrm{g}}$ to $T_{m}<T_{\mathrm{g}}$ is governed by

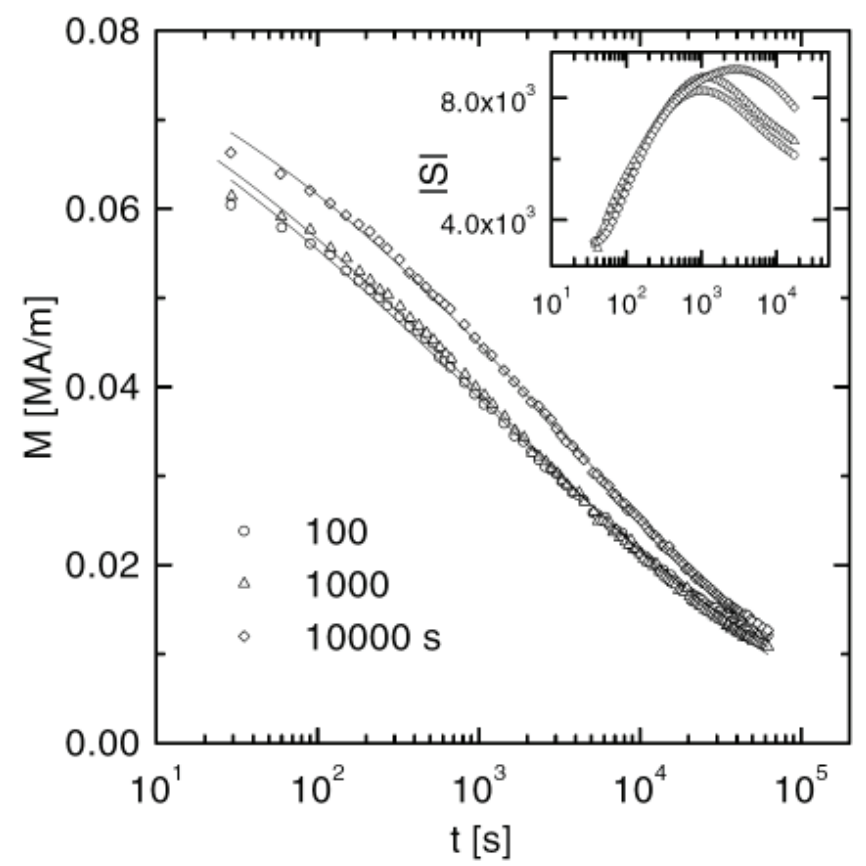

Figure 1. Relaxation curves of $M^{\mathrm{TRM}}$ at $40 \mathrm{~K}$ after wait times $t_{w}=10^{2}, 10^{3}$ and $10^{4} \mathrm{~s}$ after the sample has been cooled in a field of $\mu_{0} \mathrm{H}=0.4 \mathrm{mT}$. The inset shows the corresponding relaxation rates. The solid curves are fits to equation (1). 
the growth of equilibrium domains. The typical domain size after a time $t_{w}$ at a constant temperature $T<T_{\mathrm{g}}$ is

$$
R\left(T, t_{w}\right) \propto\left(\frac{T \ln \left(t_{w} / \tau^{*}\right)}{\Delta(T)}\right)^{1 / \psi}
$$

where $\tau *$ is the relaxation time of an individual magnetic moment, $\Delta(T)$ sets the free energy scale of the barriers and $\psi$ is a barrier exponent. The same model also predicts that the excitation of droplets due to a weak magnetic field step applied at $t=0$ occurs on length scales

$$
L(T, t) \propto\left(\frac{T \ln \left(t / \tau^{*}\right)}{\Delta(T)}\right)^{1 / \psi}
$$

within the domain walls which remain after waiting time $t_{w}$. Since both $L(T, t)$ and $R\left(T, t_{w}\right)$ grow with the same logarithmic rate, the relevant droplet excitations becomes comparable to the actual domain size at timescales $\ln t \approx \ln t_{w}$. For $\ln t \ln t_{w}$ the droplet excitations occur mainly within equilibrated regions, while for $\ln t \ln t_{w}$ excitations occur on length scales of the order of the growing domain size. Since domain walls are involved, a non-equilibrium response will result. Hence, the crossover from equilibrium to non-equilibrium dynamics occurs for $\ln t_{w} \approx \ln t$. This is seen as a peak in the relaxation rate $S(t)=(1 / H) \partial M / \partial \ln t$ versus $\ln t$ curves.

Figure 2 shows the relaxation of $M^{\mathrm{TRM}}$ at $T_{m}=35(1)$ and $27 \mathrm{~K}(2)$, with additional negative temperature cycles following protocol (2). When temporarily cooling by $T=-8$ and $-7 \mathrm{~K}$, respectively, no relaxation is observed in either case. After heating, again, to $T_{m}$, the previous relaxation continues as evidenced by shifting the timescale (solid symbols). When comparing the two cases it is conjectured that the quasi-equilibrium state reached when cooling the sample from 35 to $27 \mathrm{~K}$ cannot simply be assigned to the blocking of particle magnetic moments. As can be seen in the inset

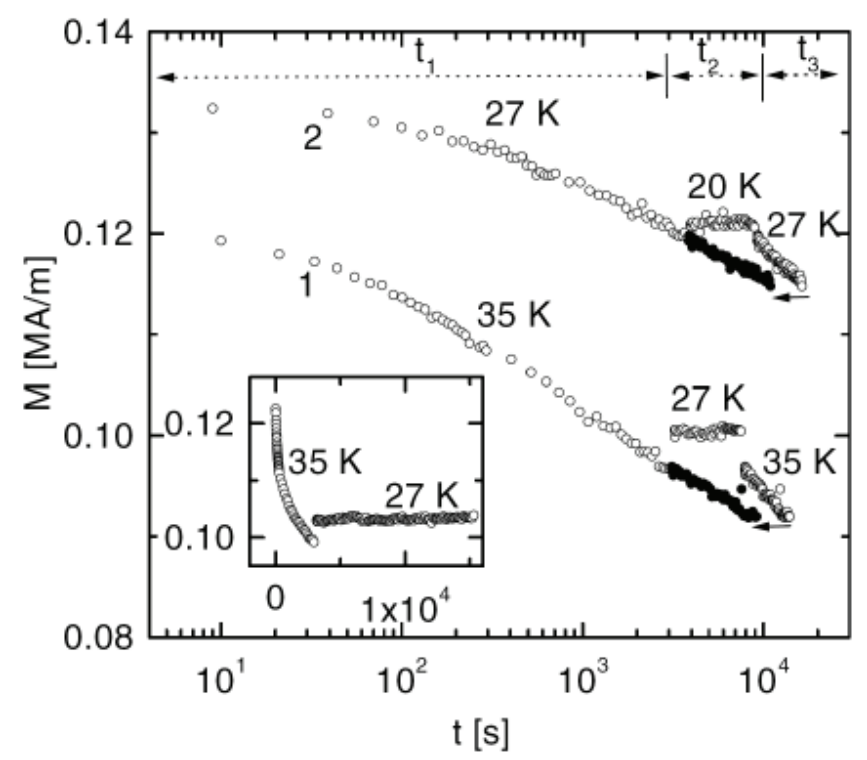

Figure 2. Relaxation curves of $M^{\mathrm{TRM}}$ after field cooling with $\mu_{0} \mathrm{H}=0.4 \mathrm{mT}$ at $(1) T_{m}=35 \mathrm{~K}(t<3000 \mathrm{~s}), 27 \mathrm{~K}(3000<t<$ $8000 \mathrm{~s})$ and $35 \mathrm{~K}(8000<t<14000 \mathrm{~s})$ and (2) $T_{m}=27 \mathrm{~K}(t<4000 \mathrm{~s}), 20 \mathrm{~K}(4000<t<9000 \mathrm{~s})$ and $27 \mathrm{~K}(9000<t<17000$ $\mathrm{s})$. The data referring to the last time intervals have been replotted against $t-8000 \mathrm{~s}$ as solid circles as indicated by arrows. The inset shows the relaxation cycle at $T_{m}=35 \mathrm{~K}(t<3000 \mathrm{~s})$ followed by a prolonged period $(3000<t<15000 \mathrm{~s})$ at $27 \mathrm{~K}$. 


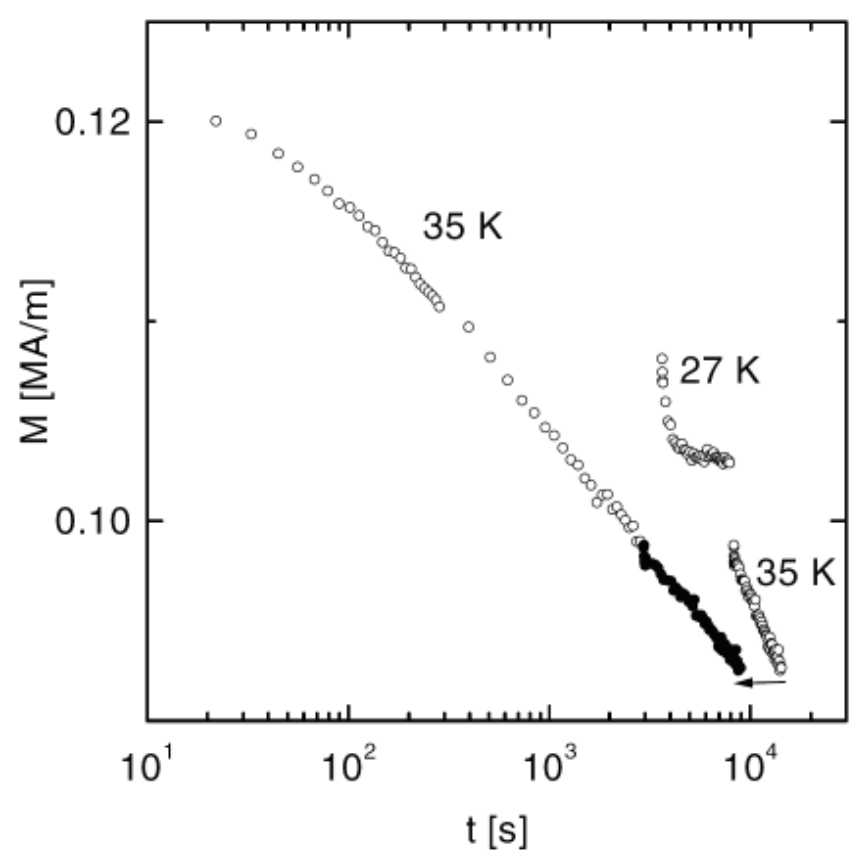

Figure 3. Relaxation curves of $M^{\mathrm{TRM}}$ after field cooling with $\mu_{0} \mathrm{H}=0.4 \mathrm{mT}$ at $T_{m}=35 \mathrm{~K}(t<3,000 \mathrm{~s}), 27 \mathrm{~K}(3,000<t<$ $8,000 \mathrm{~s})$ and $35 \mathrm{~K}(8,000<t<14,000 \mathrm{~s})$; the latter data have been replotted against $t-5,000 \mathrm{~s}$ (solid circles). Within the time interval $3,000<t<3,300 \mathrm{~s}$ a field of $0.4 \mathrm{mT}$ was applied.

to figure 2 , the relaxation involving $T_{m}=35 \mathrm{~K}$ is completely suppressed at $27 \mathrm{~K}$ even for prolonged periods, $t_{2} \approx 1.2 \times 10^{4} \mathrm{~s}$.

The reason can be provided within the framework of the droplet model as follows. It predicts that a droplet excitation, $L$, is associated with an energy barrier $B \propto L^{\psi}$ that must be surmounted by thermal activation. For the droplets to be active, the condition $F_{L} \leq k_{B} T$ should be satisfied, where $F_{L}$ is the free energy gain associated with the formation of a droplet of size $L$. Since the thermal activation process becomes slower as the temperature is lowered within the spin-glass regime, the restarted domain growth at $T_{m}-\Delta T$ cannot proceed and the domains cannot become larger than the overlap length, $l_{\Delta \mathrm{T}}$, which is a measure of the length scale below which equilibrium exists at both $T$ and $T-\Delta T$. It should be noted that $M^{\mathrm{TRM}}$ comes back to the level it reached before cooling, when the temperature returns to $T_{m}$ after temporary cooling. The solid circles show the data taken during $t_{3}$ shifted by $t_{2}$, the time spent at $T_{m}-\Delta \mathrm{T}$, along the timescale. It is found that the relaxation exactly continues the previous curve. In other words, the relaxation before temporary cooling is retrieved on returning to the measurement temperature.

On the other hand, the hierarchical picture [10] predicts the existence of a low-temperature phase with a large number of nearly degenerate states separated by finite barriers, $(T)$. Hammann et al. [13] have studied the variation of $\partial \Delta(T) / \partial T$ versus $T$ and $\partial \Delta(T) / \partial T$ versus $\Delta$ and found that $\partial \Delta(T) / \partial T$ depends only on the particular value of $\Delta(T)$ and not on the temperature. They have shown that the finite barriers between the metastable states increase very steeply with decreasing temperature. An extrapolation suggests a divergence at lower temperatures. They have also suggested that in temperature cycles, lowering the temperature splits the metastable states into a large 
number of new states. These new states merge again when the temperature is raised back. In this scenario, our experimental observations can be interpreted using the fact that intermediate cooling leads to a divergence of the energy barriers. As a consequence, the probed metastable states become pure states at the lower temperature. Thus, no new ageing is observed during temporary cooling. The system recovers the previous state when the temperature is raised back, thus yielding a memory effect.

In Figure 3 we show the relaxation of $M^{\mathrm{TRM}}$ in a similar temperature cycle involving $T_{m}=35 \mathrm{~K}$ and $\Delta T=-8 \mathrm{~K}$ following protocol (2), but this time with an applied field step of $0.4 \mathrm{mT}$ for a duration of $300 \mathrm{~s}$ just after reaching $T_{m}-\Delta T$. The relaxation is recorded after switching off the field to zero. Although the system relaxes at $T_{m}-\Delta T$, this has no effect on retrieving the initial state after returning to $T_{m}$. This behavior, of course, accords with both the droplet and the hierarchical picture. In the droplet model, the field step activates the growth of the domains, while in the hierarchical picture the free energy landscape becomes fine grained again, thus giving rise to the intermediate relaxation.

Figure 4 shows the relaxation involving $T_{m}=35 \mathrm{~K}$ and small temperature cycles of $\Delta T=-1$ (1), -2 (2) and $-3 \mathrm{~K}(3)$. The system continues to relax at $T=34$ and $33 \mathrm{~K}$, although at a slower rate during temporary cooling. Hence, on returning to $T_{m}=35 \mathrm{~K}$ the previous magnetic state cannot be retrieved. This is at variance with the situation after pulse cooling with $\Delta T=-3 \mathrm{~K}$, where a complete memory effect is observed. Obviously a specific temperature window exists within which the memory effect fails. This fact has previously been stated for conventional spin glasses [16]. The observation, of course, accords with both models. In the droplet model the statistical overlap of the droplet size distributions and in the hierarchical model the transitions over finite barriers can explain the loss of memory effect.

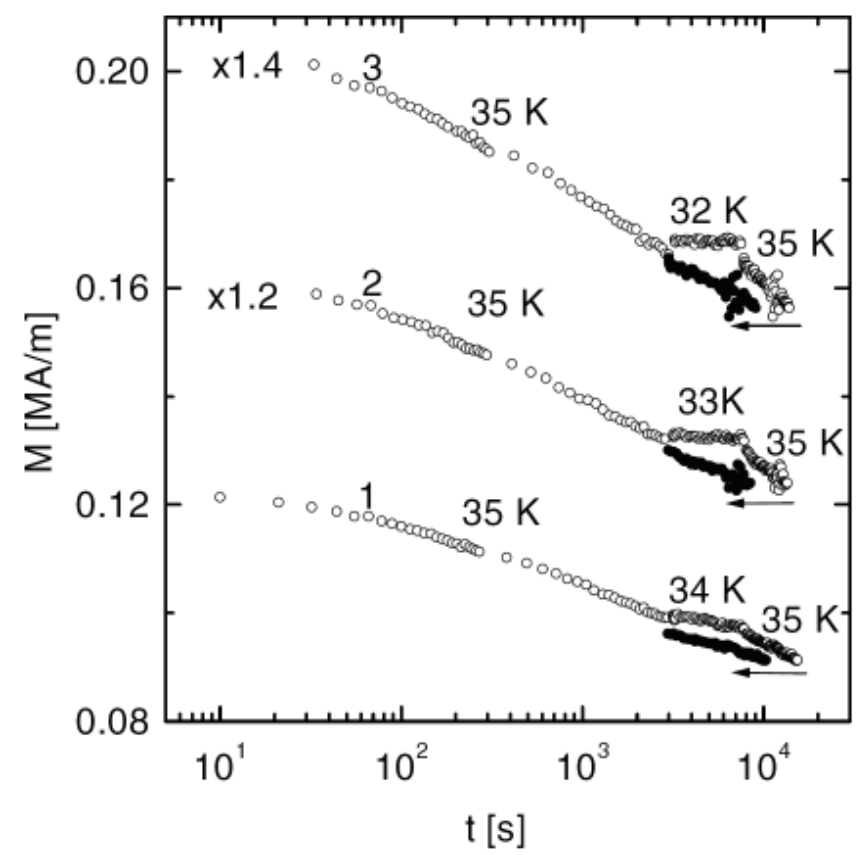

Figure 4. Relaxation curves of $M^{\mathrm{TRM}}$ after field cooling with $\mu_{0} H=0.4 \mathrm{mT}$ at (1) $T_{m}=35 \mathrm{~K}(t<3,000 \mathrm{~s}), 34 \mathrm{~K}(3,000<$ $t<8,000 \mathrm{~s})$ and $35 \mathrm{~K}(8,000<t<14,000 \mathrm{~s})$, (2) $T_{m}=35 \mathrm{~K}(t<3,000 \mathrm{~s}), 33 \mathrm{~K}(3,000<t<8,000 \mathrm{~s})$ and $35 \mathrm{~K}(8,000<t<$ $14,000 \mathrm{~s})$ and (3) $T_{m}=35 \mathrm{~K}(t<3,000 \mathrm{~s}), 32 \mathrm{~K}(3,000<t<8,000 \mathrm{~s})$ and $35 \mathrm{~K}(8,000<t<14,000 \mathrm{~s})$. All data sets obtained within $8,000<t<14,000 \mathrm{~s}$ have been replotted against $t-5,000 \mathrm{~s}$ (solid circles). 
Figure 5 shows the relaxation at subsequent positive and negative temperature cycles in one experiment. The most important observation is an asymmetric behavior in the positive cycle, where a faster relaxation is encountered during temporary heating, but is suppressed when returning to $T_{m}$ $=27 \mathrm{~K}$. Since the droplet model predicts a symmetric behavior about temperature changes, our observations contradicts the droplet picture. However, the observed results can be explained by the asymmetric variation of the free energy surface with temperature changes as proposed in the hierarchical picture. Due to an increase in temperature, the barriers have been lowered at $35 \mathrm{~K}$, thus enabling processes between renewed states, which were not accessible at $27 \mathrm{~K}$. Hence, a faster relaxation is observed upon intermediate heating.

It should be mentioned that the results reported here for positive and negative temperature cycles are not due to the specific temperatures. This has actually been checked at various temperature steps.

\section{Conclusions}

We studied the dynamics of a nanoparticle system exhibiting dipolar interaction in terms of the time and temperature dependence of the thermoremanent magnetization, $M^{\mathrm{TRM}}$. Below $T_{\mathrm{g}}$, waittime-dependent ageing phenomena are observed. They clearly exclude a model simply involving dynamic heterogeneity of uncoupled superspins with a wide distribution of activation energies. Asymmetric properties are observed for negative and positive temperature cycles. While the results of positive temperature cycles can be explained within the framework of both the droplet and the hierarchical model of spin glasses, the results for negative cycles can only be understood within

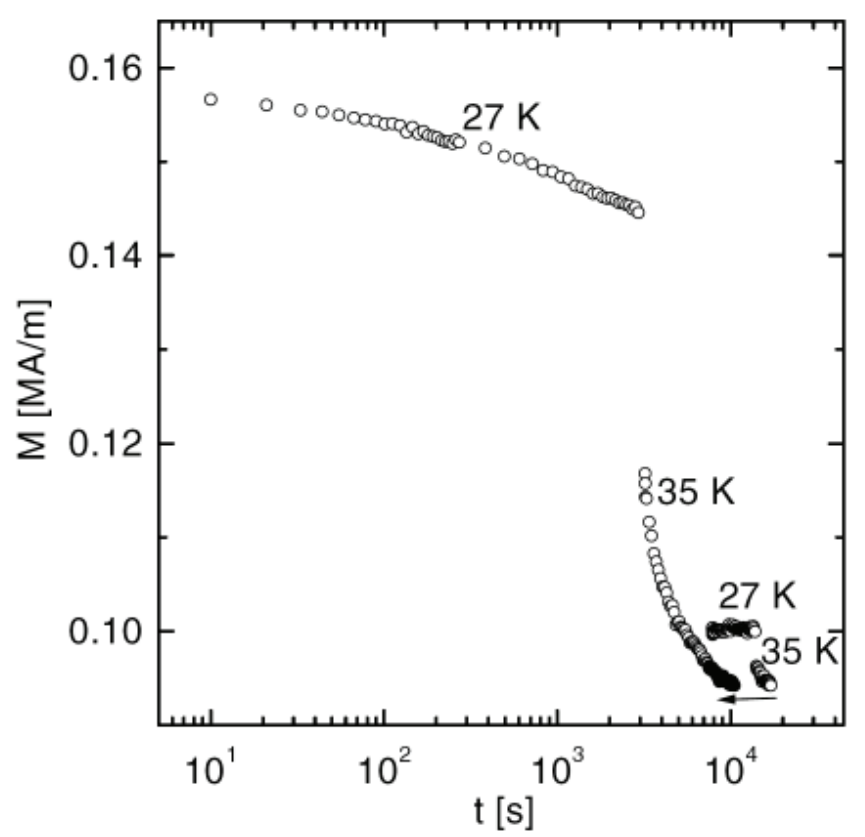

Figure 5. Relaxation curves of $M^{\mathrm{TRM}}$ after field cooling with $\mu_{0} H=0.4 \mathrm{mT}$. The positive cycle involves $T_{m}=27 \mathrm{~K}(t<$ $3,000 \mathrm{~s}), 35 \mathrm{~K}(3,000<t<9,000 \mathrm{~s})$ and $27 \mathrm{~K}(9,000<t<15,000 \mathrm{~s})$. The negative cycle involves $T_{m}=35 \mathrm{~K}(3,000<t<$ $9,000 \mathrm{~s}), 27 \mathrm{~K}(9,000<t<15,000 \mathrm{~s})$ and $35 \mathrm{~K}(15,000<t<18,000 \mathrm{~s})$. The latter data have been replotted against $t-6,000$ $\mathrm{s}$ (solid circles). 
the hierarchical model. It seems, hence, that the hierarchical model explains the experiments better than the droplet one. Presumably this is a consequence of the long-range character of the dipolar interactions involved in the SSG ordering process. They apparently preclude perfect local ordering as required within the framework of the droplet picture.

\section{Acknowledgments}

We gratefully acknowledge enlightening discussions with Professor P. Nordblad. Thanks are due to DFG (Graduate School "Structure and Dynamics of Heterogeneous Systems"), DAAD (Germany), CRUP and PRAXIS XXI (Portugal) for financial support.

\section{References}

[1] Dormann, J. L., Fiorani, D., and Tronc, E. 1997 Adv. Chem. Phys. 98283

[2] Jonsson, T., Mattsson, J., Djurberg, C., Khan, F. A., Nordblad, P., and Svedlindh, P. 1995 Phys. Rev. Lett. 754138

[3] Djurberg, C., Svedlindh, P., Nordblad, P., Hansen, M. F., Bodker, F., and Morup, S. 1997 Phys. Rev. Lett. 795154

[4] Mamiya, H., Nakatani, I., and Furubayashi, T. 1999 Phys. Rev. Lett. 824332

[5] Petracic, O., Kleemann, W., Binek, Ch., Kakazei, G. N., Pogorelov, Yu. G., Sousa, J. B., Cardoso, S., and Freitas, P. P. 2002 Phase Transitions 7573

[6] Kleemann, W., Petracic, O., Binek, Ch., Kakazei, G. N., Pogorelov, Yu. G., Sousa, J. B., Cardoso, S., and Freitas, P. P. 2001 Phys. Rev. B $\mathbf{6 3} 134423$

[7] Sahoo, S., Petracic, O., Binek, Ch., Kleemann, W., Sousa, J. B., Cardoso, S., and Freitas, P. P. 2002 Phys. Rev. B $\mathbf{6 5} 134406$

[8] Jönsson, P., Hansen, M. F., and Nordblad, P. 2000 Phys. Rev. B 611261

[9] Jonason, K., Vincent, E., Hammann, J., Bouchaud, J. P., and Nordblad, P. 1998 Phys. Rev. Lett. 813242

[10] Bouchaud, J. P., Cugliandolo, L. F., Kurchan, J., and Mézard, M. 1997 Spin Glasses and Random Fields (Series on Directions in Condensed Matter Physics vol. 12) ed A P Young (Singapore: World Scientific) pp 161-223

[11] Fisher, D. S. and Huse, D. A. 1988 Phys. Rev. B 38373 ;

Fisher, D. S. and Huse, D. A. 1988 Phys. Rev. B 38386

[12] Andersson, J. O., Djurberg, C., Jonsson, T., Svedlindh, P., and Nordblad, P. 1997 Phys. Rev. B 5613983

[13] Hammann, J., Lederman, M., Ocio, M., Orbach, R., and Vincent, E. 1992 Physica A 185278

[14] Kakazei, G. N., Pogorelov, Yu. G., Lopes, A. M. L., Sousa, J. B., Cardoso, S., Freitas, P. P., Pereira de Azevedo, M. M., and Snoeck, E. 2001 J. Appl. Phys. 904044

[15] Svedlindh, P., Granberg, P., Nordblad, P., Lundgren, L., and Chen, H. S. 1987 Phys. Rev. B 35268

[16] Nordblad, P. and Svedlindh, P. 1997 Spin Glasses and Random Fields (Series on Directions in Condensed Matter Physics vol. 12) ed A. P. Young (Singapore: World Scientific) p. 24 